\title{
Rod and cone activity in patients with dominantly inherited retinitis pigmentosa: comparisons between psychophysical and electroretinographic measurements
}

\author{
G. B. ARDEN, R. M. CARTER, C. R. HOGg, D. J. POWEll, W. J. K. ERNST, \\ G. M. CLOVER, A. L. LYNESS, AND M. P. QUINLAN
}

From the Electrodiagnostic Clinic, Moorfields Eye Hospital, City Road, London, EC1V 2PD

SUMMARY Extended electroretinographic (ERG) testing has been carried out in a series of patients with retinitis pigmentosa, dominantly inherited. In 36 of 57 cases only cone b waves were evoked. In 20 of these, psychophysical tests showed only cones mediated vision (Massof class I), while in 16 static scotopic perimetry demonstrated residual rod function (class II). In the remaining cases, where rod ERGs were seen, the light intensities required to evoke responses were not greatly elevated. A computer model was constructed to relate psychophysical threshold measurements to ERG data. This analysis of the results suggests that in one subgroup of patients the scotopic ERG is smaller than expected from the losses of visual field and that in another the psychophysical elevation of rod visual threshold is greater than expected from ERG measurements.

It is accepted that patients who are classified as suffering from retinitis pigmentosa (RP) may have one of a number of conditions, which may differ in severity, clinical manifestations, modes of inheritance, and rate of progression. ${ }^{1-5}$ Several recent investigations into autosomal dominant RP have subcategorised the condition. In some patients (class I) careful perimetric testing shows profound loss of rod function, while increase in the cone threshold is less marked; by contrast, in other patients, rod and cone threshold elevation may be approximately equal (class II). ${ }^{67}$ In a number of patients with mild RP, loss of rod sensitivity has been related to the reduction in the rhodopsin content of the retina, ${ }^{89}$ and in many cases it has been found that the reduction in the light-absorbing power of the rods totally accounts for the mild night blindness. An implication of this finding is that, when completely dark adapted, the photoreceptors and other visual neurones have normal signal transmission properties. However, when modulation sensitivity is measured by a flickering light of suprathreshold intensity, abnormalities may be detected. ${ }^{10}$

Correspondence to Professor G. B. Arden.
This paper is concerned with patients with autosomal dominant RP. We have tried to relate the ERG findings to parallel psychophysical measurements. One reason for doing so is to determine whether the ERG can distinguish between the subdivision of Massof's classes I and II proposed on the basis of psychophysical tests. ${ }^{67}$ We have also obtained rod ERGs to differing intensities of stimulus, and in addition to measuring the amplitude of the $b$ wave we have defined working range of the ERG by a parameter, ' $\mathrm{K}$ ', ${ }^{112}$ defined as the light required to produce a half maximal rod $b$ wave. The patients have differing degrees of loss of sensitivity and visual field, and we have attempted to discover whether any simple relationship exists between such losses and the degree of abnormality of the ERG. In order to do this quantitatively we have made a computer model.

\section{Methods}

All patients were given thorough clinical examinations in the genetic clinic, where their affected and unaffected relatives were interviewed, to determine the mode of inheritance. 


\section{ELECTRORETINOGRAPHY}

The techniques have been fully described. ${ }^{12}$ In brief, gold foil electrodes were employed so that prolonged recordings were possible. The ERGs were evoked by a variety of different ganzfeld stimuli, so that rod sensitivity and the relative amplitudes of rod and cone generated responses could be compared. In those patients where rod responses could be detected the 'long protocol' previously described was usually followed, but in some cases we used a shorter version, designed so that rod $b$ waves of 20,60 , and $100 \%$ maximum were elicited. The rod b wave amplitude/ light intensity relationship was fitted to curves of the form ${ }^{12}$ (equation 1)-

$$
\mathrm{V}=\mathrm{V}_{\max }(\mathrm{I} /(\mathrm{I}+\mathrm{K}))
$$

VISUAL FIELD EXAMINATIONS

The visual fields were measured with a calibrated Goldmann perimeter, using I,4 and IV,4 targets. The relative and absolute sensitivities of many different portions of the retina were measured by an automated perimeter. ${ }^{13}$ This consisted of a Lister perimeter in which the target was replaced with both red and green light-emitting diodes (LEDs). These were energised by very brief pulses, the frequency of which could be varied from $50 \mathrm{~Hz}$ to $0.35 \mathrm{MHz}$. Light intensity was altered by changing the pulse frequency (coarse control) or the current flowing through the LEDs (fine control). The light intensity could be varied in steps of $0 \cdot 1 \log$ unit over a range of $5 \log$ units. The perimeter was driven by a computer, and darkadapted absolute visual thresholds were determined at various parts of the visual field. A greater loss of sensitivity with the green than with the red test light indicates a disorder having a greater effect on the scotopic than the photopic system. Thresholds were tested (in most patients) at $5^{\circ}$ intervals along 4 visual meridians, which were chosen to obtain the maximal amount of information about the patients' condition and to reveal the extent of field constriction, presence of annular scotomata, etc.

\section{Results}

The series consists of 57 patients for whom ERG, psychophysical, genetic, and clinical data are available. Patients were excluded only if the data were incomplete, or technically unreliable.

In 21 cases the ERGs recorded to relatively dim flashes of blue light had a slowly rising, rounded waveform, typical of responses generated by the

Table 1 Subdivision of patients by ERG results

\begin{tabular}{|c|c|c|c|c|}
\hline & \multicolumn{2}{|c|}{ Group A } & \multicolumn{2}{|c|}{ Group B } \\
\hline & Mean & $S D$ & Mean & $S D$ \\
\hline Number of cases* & 36 & & 21 & \\
\hline Age & $\begin{array}{l}40 \\
13-79\end{array}$ & & $\begin{array}{l}40 \\
22-71\end{array}$ & \\
\hline $\begin{array}{l}\text { Blue light } \\
\mathrm{V}_{\max } \mu \mathrm{V} \\
\text { peak time, ms* } \\
\text { within normal limit }\end{array}$ & $\begin{array}{c}11 \cdot 5 \\
61 \cdot 5 \\
0\end{array}$ & $\begin{array}{l} \pm 8 \cdot 6 \\
\pm 17 \cdot 3\end{array}$ & $\begin{array}{r}183 \\
64 \\
7\end{array}$ & $\begin{array}{l} \pm 116 \\
\pm 8 \cdot 5\end{array}$ \\
\hline $\begin{array}{l}\text { Red light } \\
\mathrm{V}_{\max } \mu \mathrm{V} \\
\text { peak time, ms* } \\
\text { within normal limit }\end{array}$ & $\begin{array}{c}12 \cdot 5 \\
55 \\
0\end{array}$ & $\begin{array}{l} \pm 12 \cdot 5 \\
\pm 9 \cdot 3\end{array}$ & $\begin{array}{r}91 \\
50 \\
4\end{array}$ & $\begin{array}{l} \pm 62 \\
\pm 6\end{array}$ \\
\hline $\begin{array}{l}\text { White light } \\
V_{\max } \mu \mathrm{V} \\
\text { peak time, ms* } \\
\text { within normal limit }\end{array}$ & $\begin{array}{l}17 \cdot 5 \\
56 \\
0\end{array}$ & $\begin{array}{l} \pm 17 \cdot 4 \\
\pm 10\end{array}$ & $\begin{array}{r}230 \\
50 \\
10\end{array}$ & $\begin{array}{l} \pm 155 \\
\pm 6\end{array}$ \\
\hline $\begin{array}{l}\text { Flicker } \\
\text { \% of single flash } \\
\text { ERG peak time, ms } \\
\text { within normal limit }\end{array}$ & $\begin{array}{r}46 \\
39 \\
0\end{array}$ & $\begin{array}{l} \pm 16 \\
\pm 4 \cdot 6\end{array}$ & $\begin{array}{l}20 \\
32 \\
10\end{array}$ & $\begin{array}{l} \pm 17 \\
\pm 4.9\end{array}$ \\
\hline $\begin{array}{l}\text { Goldmann field size } \\
\text { (mean outer margin, degrees) } \\
\text { within normal limits }\end{array}$ & $\begin{array}{r}18 \\
0\end{array}$ & \pm 13 & $\begin{array}{r}33 \\
3\end{array}$ & \pm 15 \\
\hline
\end{tabular}

* Note those cases where response absent neglected in calculations. 
rods. The amplitudes varied considerably, as did the light intensities required to elicit maximal responses. If red light was used as a stimulus, a double-peaked ERG was seen in most of these patients' records, though in those cases where the retina was relatively insensitive to blue light and the response was small the second slow peak might be absent. In the remaining 36 cases no ERG was evoked by dim blue light flashes, but, if the light was made more intense, in all but 6 cases a b wave of more than $5 \mu \mathrm{V}$ was seen. However, it was small and rose to and fell rapidly from the peak. In short it resembled a cone response. There was also a shift in relative spectral sensitivity. Fig. 2 shows that the slow rod responses evoked by the red flash are matched in amplitude by a weak blue

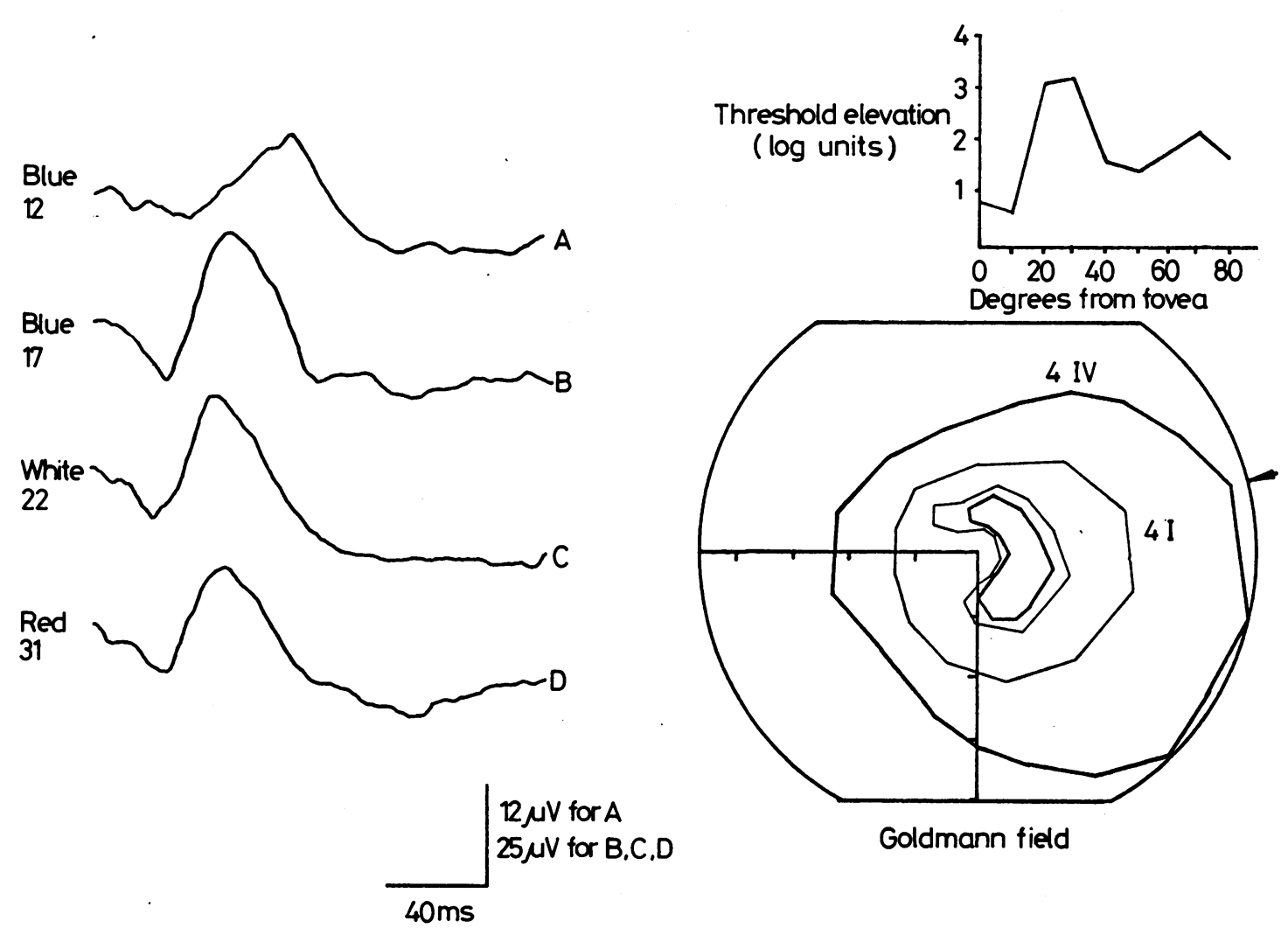

Fig. 1 Records from a patient classified as belonging to group A. On the left are some of the ERGs obtained. The record numbers shown to the left of the traces refer to the sequence of stimuli detailed in the appendix to Arden et al..$^{12}$ Note the responses are small, and fast, red and blue stimuli evoking similar waveforms, associated with cone responses. One inset on the right shows the Goldmann perimeter result for the right eye. The graticule marks on the figure represent $20^{\circ}$ intervals. Test targets of 2 sizes were used to obtain the fields, as indicated in the figure. Note that there is the beginning of an annular scotoma in the temporal field. The arrow shows a meridian along which absolute visual threshold was determined, at 5 intervals, and the upper inset summarises these findings. In the graph the ordinate is loss of rod sensitivity, and the abscissa retinal eccentricity. Note the rise of visual threshold in the area of the scotoma disclosed by Goldmann perimetry. This patient has unrecordable rod ERGs and therefore belongs to group A. However, it can be seen that the elevation of visual threshold over most of the field is less than 2 log units, so that some psychophysical rod function was preserved. The discrepancy between ERG and threshold estimates of rod function place this patient in group A2. Compare these results with those of Fig. 2, where rod ERGs are easily demonstrable, despite the field being smaller. Patient 9890 , male, aged $49 \mathrm{yr}$. 

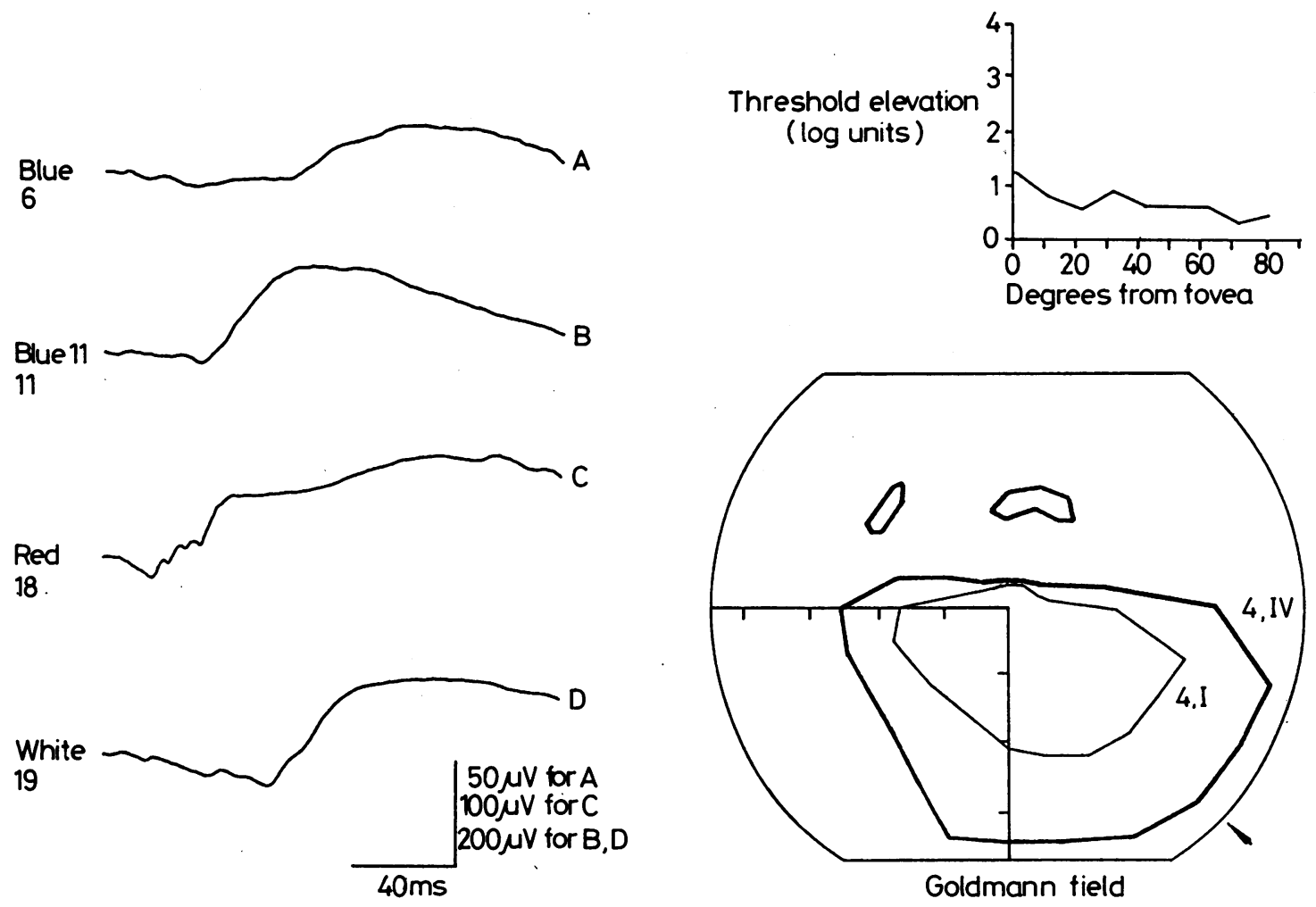

Fig. 2 Records from a patient classified as belonging to group $B$. The records shown are to be compared with those in Fig. 1 . Slow rod ERGs can be seen with dim blue light, and red light produces a double-peaked ERG. The photopic field is reduced, but no perimetric determination was 'overrange', showing rods continue to function through a large portion of the visual field, though their sensitivity is reduced. Large rod ERGs are obtained. Note the difference in the calibration bars for this and the preceding figure. Patient number 10822, female aged 65 yr (see Fig. 8).

abnormality which is significantly greater in group $A$. Note also that for $B$ there is a larger reduction of the response when flickering light is used as a stimulus, indicating that in that group there is a contribution of rods to the single-flash ERG. Another difference between the groups is that in $B$ the Goldmann fields were larger.

Figs. 1 and 2 show illustrative records obtained from patients in groups $A$ and $B$. Note the difference in the calibration bar voltages for the ERGs, and the differences in ERG waveform. The red light ERG in Fig. 1 is $20 \mu \mathrm{V}$ in amplitude, about half the size of the rapid initial $b_{1}$ or $x$ wave to the corresponding stimulus in Fig. 2. The size of the photopic field, determined by the Goldmann perimeter, is larger in Fig. 1 than in Fig. 2, and in the former case an annular scotoma is developing. The arrowheads at the borders of the representation of the Goldmann field charts show the meridia at which the scotopic threshold determinations illustrated were made. In Fig. 1 there is a great loss of sensitivity in the region of the scotoma, but otherwise the threshold evelvation ranges from half to under $2 \log$ units. In Fig. 2 the threshold elevation is less, ranging from above 1 log unit in the central field to half a log unit in the extreme periphery.

Comment. Both young and old patients are found in groups $A$ and $B$, which implies that the rod $\mathrm{b}$ wave is not absent in $A$ simply because the patients, owing to advancing age, have reached an end-stage in a progressive disease. In some group $A$ cases, where the patient has tunnel vision, it is possible that the remaining functional area of retina is the relatively rod-free macular zone, and this could explain why cone but not rod ERGs are recorded. However, as Fig. 1 shows, some patients in group $A$ have well preserved photopic fields. In them rod ERGs should be seen, if rods and cones were preserved over similar areas of the retina, just as they are in the case of group $B$ patients (Fig. 2). It might be thought that rod ERGs were not seen because the rods were insensitive to light, but, as Fig. 1 shows, in a patient who has rod function over a considerable portion of the visual 
field-even though threshold is elevated by $1-2$ log units-the scotopic b wave may be totally absent, even when intense lights are used to stimulate the retina. Such observations recall the classification of Massof et al. ${ }^{67}$ who found that RP patients could be subdivided according to whether rod function was selectively reduced (class I) or rods and cones were approximately equally affected (class II), and suggest that groups $A$ and $B$ might represent different disease entities. In order to establish this, and to determine the relationship between the electrophysiological and psychophysical classifications, we require a more quantitative method of estimating the ERG expected from field and threshold data. As a first step we analysed the results from our patients to see whether there was a correspondence between ERG and psychophysical evidence of rod function.

\section{SUBDIVISION OF PATIENT GROUP WITH NO ROD ERG}

The 36 patients with no rod ERG may be divided into 2 categories. Twenty had psychophysical thresholds exclusively determined by cones $\left(A_{1}\right)$ and in 16 there was evidence of rod function $\left(A_{2}\right)$. The range of amplitudes of the (cone) ERGs is similar in the 2 subgroups, as shown in Fig. 3. There are no significant differences between any other aspect of the ERG

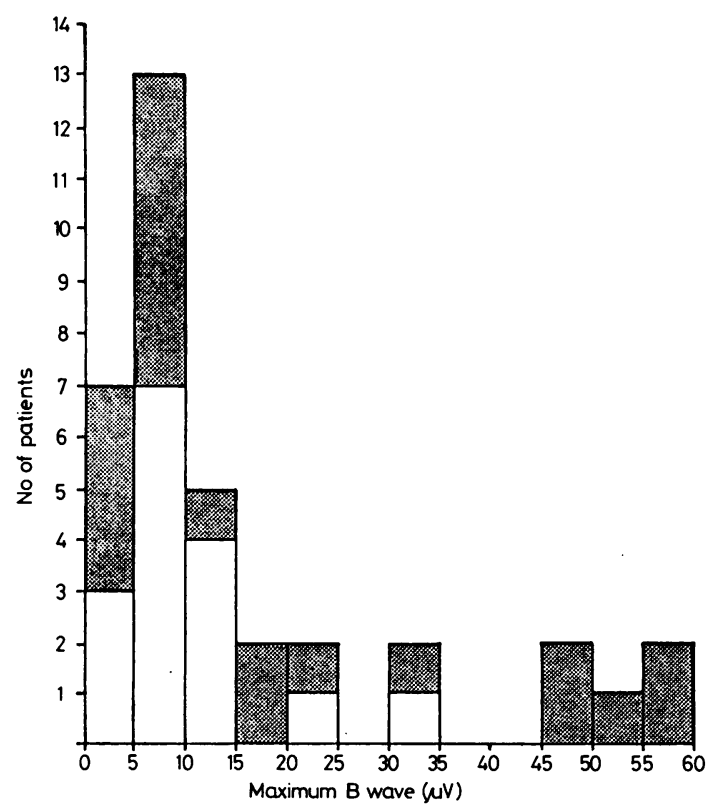

Fig. 3 Histogram showing distribution of cone $b$ wave amplitudes in patients with no rod ERG. Group $A_{1}$ (psychophysically night blind) are represented by shaded areas, and group $A_{2}$ (psychophysical evidence of rod function, but no rod ERG) by clear areas. measurements for the subgroups. Inspection of the perimetric results shows that the group $A_{2}$ patients all retained residual rod function in the central $5^{\circ}$ of the visual field, but in 7 cases only was the threshold in more peripheral regions low enough for it to be possible to determine whether rods or cones mediated vision. One of these patient's results is shown summarised in Fig. 1. In the other cases the results were less clear-cut, because the area of preservation of rods was smaller. Before one can decide whether any $b$ wave would be detected in such cases it is necessary to establish a relationship between the amplitude of the ERG and the size of the field (see below).

When the results were subjected to an analysis of variance, some statistical differences between the 2 subgroups were found. For group $A_{1}$ there is a significant correlation between age and field size, and

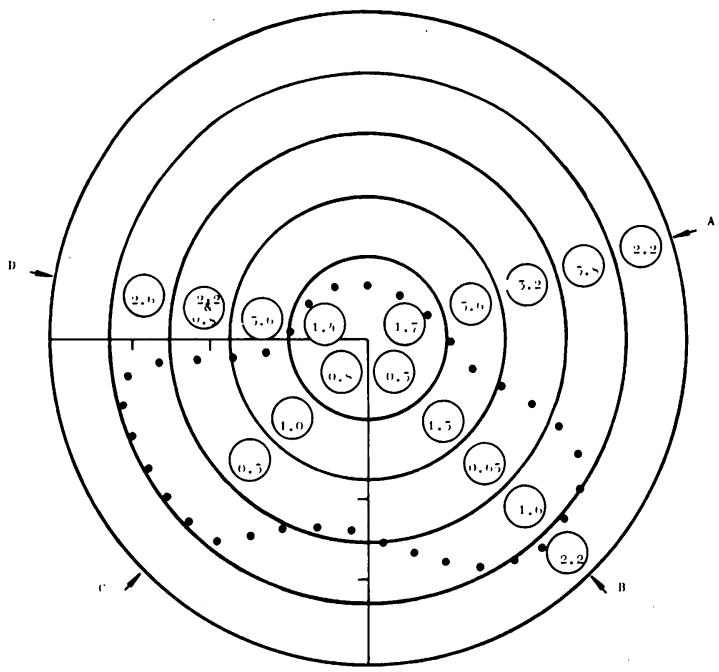

Fig. 4 Diagram to illustrate how the values of the computer model were obtained: to be read in conjunction with Table 2 . The diagram shows a perimeter chart (not from any one patient) divided into 5 concentric annuli. The horizontal and vertical crossed lines give the retinal eccentricities at $20^{\circ}$ intervals. Visual thresholds were determined along 4 meridia, $A, B, C$, and $D$ as indicated, at $5^{\circ}$ or $10^{\circ}$ intervals. In some patients different meridia were used. The average threshold elevation is indicated within the circles (except in one case-see below) and is obtained from several values. The dotted line shows the approximate outline of the visual field as determined by Goldmann perimetry. Note for annulus 3, meridian $D, 2$ values are shown. This point lies at the margin of the photopic field, and one threshold determination shows rods are active. In the computer model the value of $0 \cdot 8$ would indicate that this point produced $a b$ wave. In the adjacent region, where threshold was elevated by $2 \cdot 2$ log units, the model would assume that no $b$ wave was evoked. This leads to estimates of the value of $V_{\text {max }}$ for any one patient. The steps in the computer programme, which produced the model values are shown in Table 2. 
Table 2 Parameters used in modelling the ERG perimetric data

\begin{tabular}{|c|c|c|c|c|c|}
\hline A. Annulus number & 1 & 2 & 3 & 4 & 5 \\
\hline B. Inner and outer limits, degrees & $1-14^{1}$ & $20-35$ & $35-50$ & $50-65$ & $65-80$ \\
\hline C. Relative retinal area (percent) ${ }^{2}$ & $5 \cdot 29$ & $20 \cdot 25$ & 25.93 & $26 \cdot 86$ & $21 \cdot 64$ \\
\hline D. Corrected annular $V_{\max }$ value $(\mu \mathrm{V})^{3}$ & $21 \cdot 1$ & $80 \cdot 6$ & $103 \cdot 2$ & $106 \cdot 9$ & $86 \cdot 1$ \\
\hline E. Number of threshold measurements ${ }^{4}$ & 4 & 8 & 6 & 6 & 4 \\
\hline $\begin{array}{l}\text { F. Number of measurements showing rods determine } \\
\text { thresholds (Fig. } 4)^{5}\end{array}$ & 4 & 4 & 3 & 2 & 0 \\
\hline G. Corrected annular $V_{\max }$ value $(\mu \mathrm{V})(\mathrm{D} \times \mathrm{E} / \mathrm{F})$ & $21 \cdot 1$ & $40 \cdot 3$ & $51 \cdot 5$ & $32 \cdot 3$ & 0 \\
\hline $\begin{array}{l}\text { H. Average loss of rod sensitivity (log units) (from } \\
\text { measurements shown in F only) }\end{array}$ & $1 \cdot 1$ & $1 \cdot 25$ & $0 \cdot 65$ & $1 \cdot 6$ & - \\
\hline
\end{tabular}

Calculated increase in ' $K$ ': $1 \cdot 15 \log$ units.

Calculated $b$ wave amplitude for $I=0 \cdot 2 \mathrm{q} / \mathrm{rod}$ (normal ' $\mathrm{K}$ ' value): 23 microvolts.

Calculated $V_{\text {max }}$ : 241 microvolts.

'These values where chosen to avoid threshold measurements near the blind spot.

${ }^{2}$ Calculated from geometric considerations and Drasdo's correction. ${ }^{15}$

${ }^{3}$ Average normal b wave 398 microvolts: voltage generated by each annulus proportional to area.

${ }^{4}$ Measurements were made at $5^{\circ}$ or $10^{\circ}$ intervals: the position and number of the meridia used varied over the course of the investigation. The figures given are for exposition. For simplicity of illustration all the measurements in one annulus at one meridian are condensed into a single number in Fig. 1 (except in one case) to illustrate how, in this version of the model, estimates of $V_{\max }$ and sensitivity were obtained-see text for details.

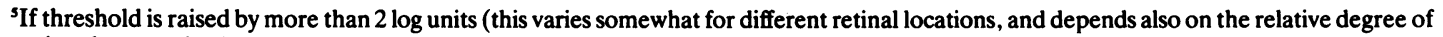
cone involvement in the particular patient) it is impossible to determine if rods or cones mediate sensation, and it is assumed no ERG is produced.

field size and $b$ wave amplitude, for both single flash and flicker. In other words, the older the patient the worse the responses. Such correlations were not found in group $A_{2}$. In that group there is a very strong correlation between psychophysical elevation of the rod threshold (averaged from perimetry values according to the model to be described below) and the reduction in the cone ERG amplitude. The fact that differing correlations are established in the 2 groups suggests that $A_{1}$ and $A_{2}$ may represent differing diseases.

\section{PATIENTS WITH ROD ERGS}

Twenty-one of the sample $(40 \%)$ are in this group $(B)$, and in all there was psychophysical evidence of functioning rods; these patients would all be included in Massof's class II. In as many cases as possible we determined the value of $\mathrm{V}_{\max }$ and of the parameter ' $\mathrm{K}$ ', which has the value of the light intensity required to evoke a half maximal rod b wave. Such calculations were possible for group $A$ patients when the ERG was of fair size, but the value of ' $K$ ' in such cases was always extremely elevated relative to the normal. This partly reflects the fact that for these patients the working range of a photopic mechanism was compared with the scotopic responses of normal subjects. In group $B$ the average value of ' $K$,' as defined in the 'Methods' section, was increased by only $0.27 \log$ unit. On the other hand previous psychophysical estimates of rod sensitivity in such patients have always revealed obvious elevation of threshold, ${ }^{14}$ and this was also true for many of the patients in this series. Thus again it appeared that there may be a discrepancy between the ERG and psychophysical data, but before such a statement can be accepted it is desirable to quantify the results.

\section{A MODEL TO RELATE ERG AND PSYCHOPHYSICAL DATA}

In making such a model there are many unknown variables, and exact correspondences between the results predicted by the model and those actually observed are not to be expected. The main purpose is to see if there are systematic differences between the predictions and the observations. We have therefore made the model as simple as possible. The following assumptions have been made.

(a) The ERG voltage produced by each small portion of the retina does not depend on the retinal eccentricity but is proportional to its area (the unit of area considered is so large that the presence of a rod-free fovea centralis does not invalidate the assumption).

(b) In patients the value of the parameter ' $\mathrm{K}$ ' for each retinal area is increased relatively to the average normal by the same factor as indicated by the 
threshold (rod) measurements made with the static perimeter.

(c) If the retinal sensitivity at any point is decreased to a level where it is not possible to determine psychophysically whether rods or cones generate the sensation, no $\mathrm{b}$ wave is produced.

(d) The measurements made with the static perimeter are representative of the sensitivity of the retina as a whole.

Fig. 4 is a diagram which represents the threshold elevations and the size of the visual field which might be obtained in one of our patients. The steps in the calculation can be followed by reference to that figure and to Table 2. The extent of the normal field of vision was split into 5 concentric annuli (row $A$ ), the limits of which are given in row $B$. The relative areas of each annulus (row C) were calculated from geometric considerations with the use of a correction described by Drasdo and Fowler. ${ }^{15}$ The relative amplitude of the maximum $b$ wave produced by each annulus (assumption $a$ ) in a normal subject is shown in row $D$. A number of threshold measurements were made in each annulus (row $E$ ), and in the patients these values were usually elevated. If threshold elevation was greater than about $2 \cdot 2 \log$ units (the exact value varied across the visual field ${ }^{13}$ ) it became impossible to decide whether the sensation was generated by rods or by cones. Such 'overrange' threshold measurements were assumed to come from regions which did not generate a rod ERG (assumption $c$-but, see below, modifications were later made to the model). The number of points in each annulus which gave a useful rod threshold (row F) determined the relative maximum ERG obtained from the annulus. The inset circles in Fig. 4 show the increases in threshold along the 4 meridia usually employed. Note that thresholds were determined at $5^{\circ}$ or $10^{\circ}$ intervals, so that the value given in each circle is an average of several determinations. In one case 2 numbers are included in the circle. This is to indicate that in that region there was an abrupt change in visual threshold. Row F in Table 2 indicates that in such a case only the individual threshold determinations which were 'within range' were used to determine $V_{\max }$. The values of $\mathrm{K}$ for all these points were then averaged, giving the value shown in row $\mathrm{H}$. From the values in rows $G$ and $H$ it was possible to calculate ERG voltages for each annulus for any light intensity, according to the formula ${ }^{112}$ -

$$
\mathrm{V}=\mathrm{V}_{\max }(\mathrm{I} /(\mathrm{I}+\mathrm{K}))
$$

for various values of $I$, with $V_{\max }$ decreased relative to the normal by the proportion of overrange threshold measurements, as described above. Similar calculations were made for each annulus, the calculated response amplitudes summed for all annuli, and rescaled for comparison with the experimentally determined ERG voltages. The calculations were carried out on a computer. Various versions of the model were constructed, embodying different assumptions, to see whether the conclusions drawn were critically dependent on the assumptions (see below).

\section{COMPARISON OF ERGS WITH COMPUTER}

PREDICTIONS FROM PERIMETRY

Amplitude predictions. Fig. 5 shows the rod b wave amplitudes (dots) in group $B$. Predicted values are shown on the abscissa and observed values on the ordinate. The continuous line drawn at $45^{\circ}$ shows the expected relationship if the model predictions were fulfilled. It can be seen that for some patients with both small and large ERGs the predictions were reasonable, but the model has a bias and tends to predict larger ERGs than are observed. The dashed lines which are drawn symmetrically about the $45^{\circ}$ line include all the cases in which the ERG was larger than predicted but exclude 7 cases (the filled circles which fall below the dashed line), where smaller than predicted ERGs were observed. The figures suggests that the patients in whom rod and cone function were both preserved (Massof class II) can be further subdivided according to the ERG results. Analysis of individual cases is given later. Additionally in 7 cases of group $A_{2}$ the area of preserved rod function was sufficient for the model to make predictions about the

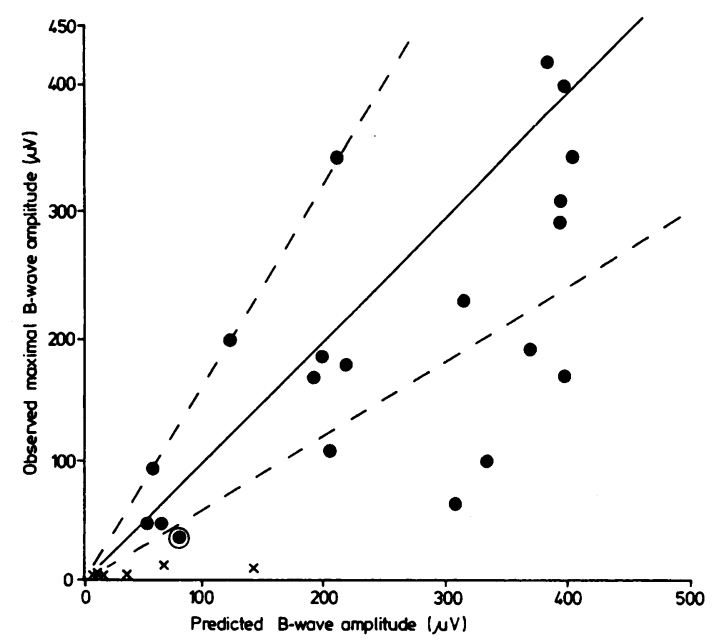

Fig. 5 ERG amplitudes in group $B$ patients (dots) and $A_{2}$ patients (crosses). Ordinate is the observed value, and abscissa, amplitude predicted from the computer model. The full line at $45^{\circ}$ is the expected locus of the points if the model's assumptions are correct. The dotted lines are drawn symmetrically about the oblique to demonstrate that the model has a bias and predicts larger ERGs than are found. 


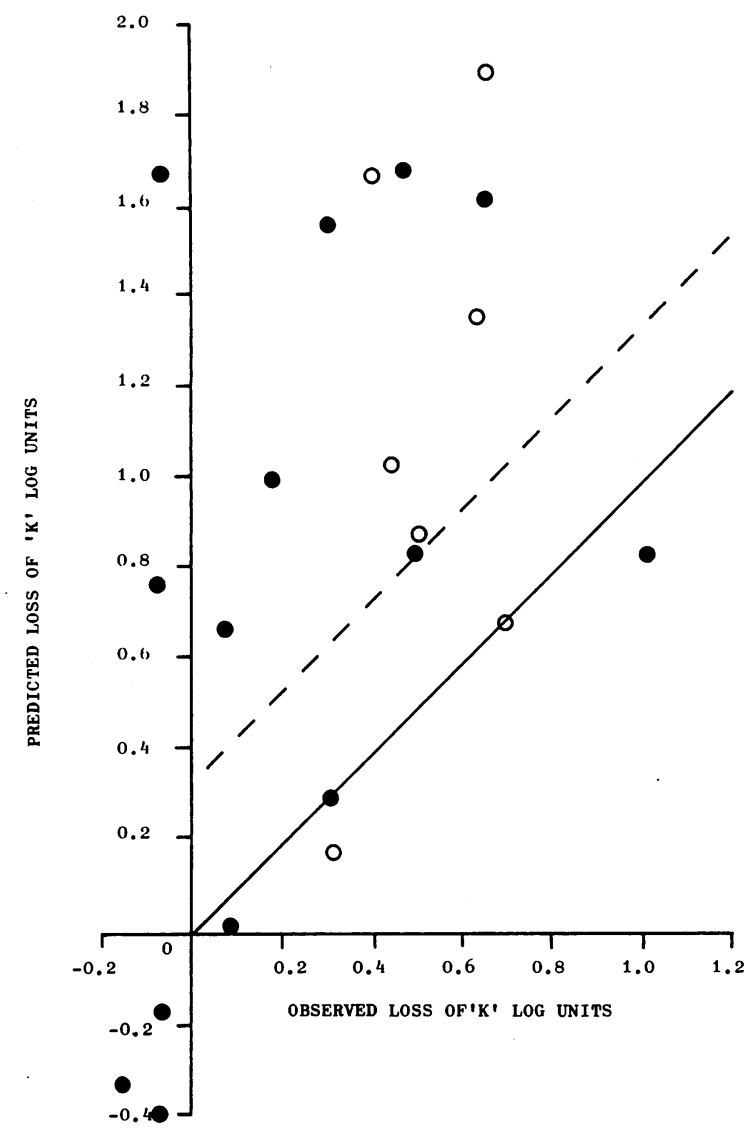

Fig. 6 Alterations in the value of ' $K$ ' for cases of dominant $R P$. All results are from patients in group $B$. The abscissa shows the observed changes in the parameter, while the ordinate gives the changes predicted by the computer model. The patient with the lowest threshold has a sensitivity $0.4 \log$ unit greater than the average normal value. A dashed line at $45^{\circ}$ projected from this point would include all the results in which the predicted value of ' $K$ ' was less than observed. The dashed line drawn in symmetry indicates the limit of experimental error. Points above and to the left show cases where the predicted increase in threshold is greater than that actually observed. The value of ' $K$ ' determined is on average increased by less than $0 \cdot 3 \log$ unit. The open circle results are those where there was the greatest discrepancy between the observed and predicted $V_{\text {max }}$.

amplitude of the rod ERG. These patients' results are indicated by the crosses in Fig. 5. In all the cases the ERG is smaller than predicted. It should be remembered that these ERGs are generated by cones, and the rod response is presumed to be totally absent, so the discrepancy between observation and the model is even larger than indicated by the symbols on the figure. In some cases the model predicts that the rod b wave will be small, but in one case (that illustrated in Fig. 1) nearly $150 \mu \mathrm{V}$ of rod b wave was predicted, and none found.

Values of the parameter ' $K$.' Fig. 6 shows the observed and predicted changes in ' $K$ ' for group $B$. There are 3 points in which predicted and observed values of $K$ are negative and one positive value near the origin. These results are obtained from members of one family, with very mild disease with delayed onset. It is possible that some of these patients have not inherited the disease, and if so these results indicate the limits of experimental error. In the other patients there was some psychophysical evidence of night blindness. However, in most patients the observed loss of ' $K$ ' was small. The oblique line shows the locus on which experimental points should fall, if the model's predictions were accurate. The dashed line shows the limit of experimental error (cf Fig. 5). No patient has an ERG with an elevation of ' $K$ ' significantly greater than predicted. In 5 cases there is reasonable agreement between the prediction and the observation. In the remaining 14 cases the points lie above and to the left of the dashed line-that is, the psychophysical threshold elevation is so great that the model predicts a larger change in ' $K$ ' than is observed. The deviations are considerable. The largest increase in the observed value of ' $K$ ' in this group is $0.7 \mathrm{log}$ unit, corresponding to a 5 -fold increase in the quantity of light required to elicit a half maximal response. However, the predicted loss of sensitivity is much greater, ranging up to almost 2 log units. The model was constructed so that it would not accept any greater loss. Overall, the predictions from the model do not fit the experimental results. There is no relationship between the ability of the model to predict the amplitude of the ERG and accuracy of the prediction of the value of ' $K$ '. The open circles of Fig. 6 are the values of ' $K$ ' obtained from the 7 patients whose ERGs amplitudes were smaller than predicted $\left(\mathrm{V}_{\max }\right.$ estimates below the lower dashed line in Fig. 5). In 3 of these, the ' $K$ ' values agree with the psychophysical estimate. The results for the group $A_{2}$ patients are not plotted in Fig. 6: they would cluster around an extension of the oblique line, at the extreme top right of the figure, and are not included because the comparison would be between observed cone sensitivity and predicted rod sensitivity.

MODIFICATIONS TO THE MODEL

Although in over half the cases the model is successful in predicting the maximal ERG response, it is less successful in predicting the value of ' $K$ '. It should be noted that the loss of amplitude is underestimated and the loss of sensitivity overestimated. The model was modified to see if betier predictions could be made. The conclusion was that this could not be done 


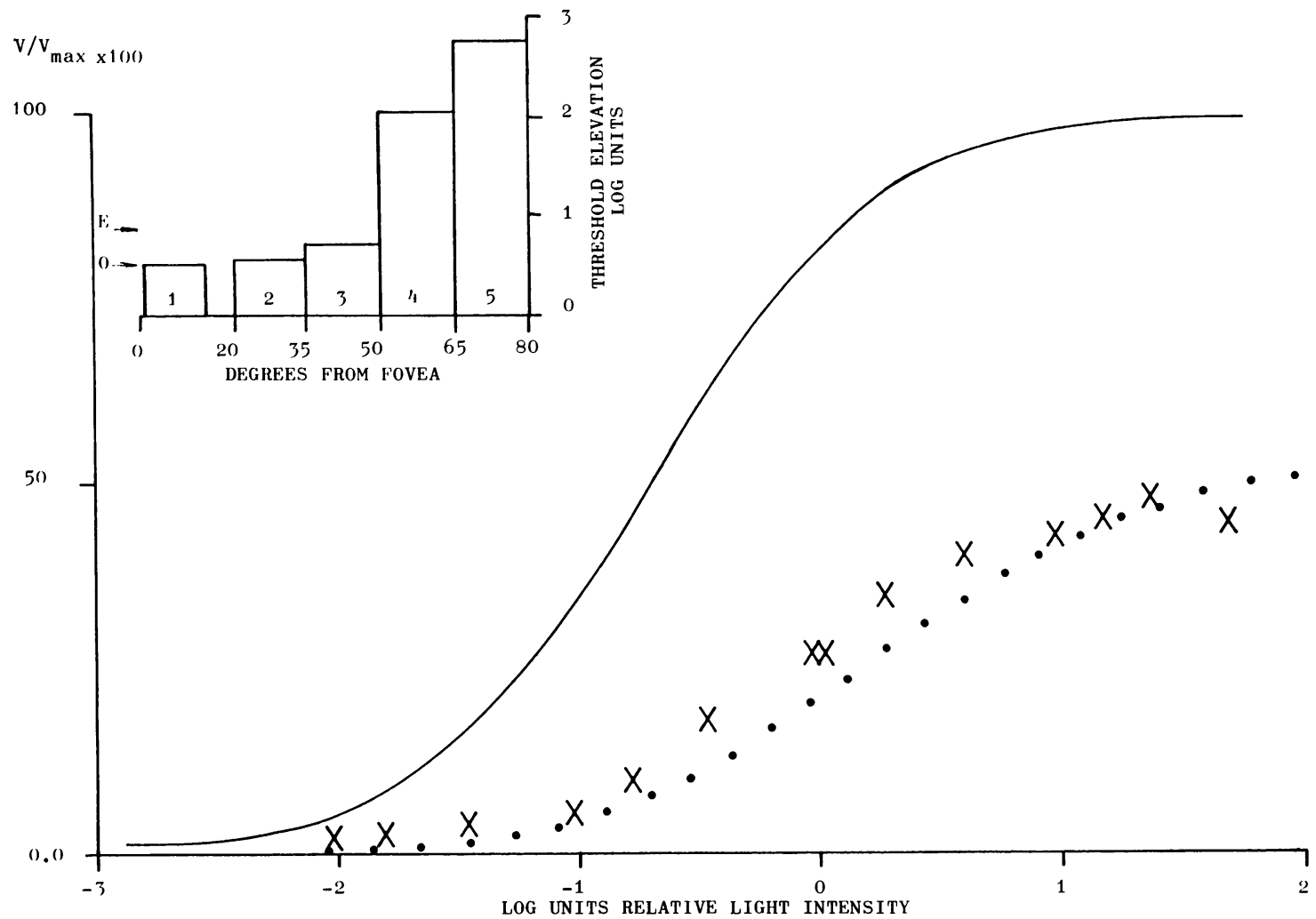

Fig. 7 Patient 10486, female aged 50, autosomal dominant RP. Comparison of the computer model and the experimental ERGs. In this and subsequent figures the continuous line is the average normal curve, $V=V_{\max }(I / I+K)$ where $V_{\max }$ is $398 \mu V$ for blue light flashes $\left(\right.$ see Arden et al. $\left.{ }^{12}\right)$. The ordinate scale shows $\left(V / V_{\max }\right) \times 100$. The dots show the prediction of the model and the crosses the results actually obtained. The amplitude of the ERGs is obtained by multiplying the ordinate value by 3.98. A summary of the psychophysical sensitivity losses is shown as an inset. There is greater threshold elevation in the peripheral field. In this patient the predicted maximal ERG voltage and the experimental value agree well, and the change in the value of ' $K$ ' given by the model is only slightly greater $(0 \cdot 8 \mathrm{log}$ unit) than that determined experimentally $(0 \cdot 5)$.

by simple changes to the assumptions. Thus, increasing the weighting for the annuli nearer the fovea will increase the predicted ERG in patients with greater field loss in the periphery, but those in whom the rod sensitivity is preserved in the far periphery will have the ERG underestimated. Both types of field loss are seen in the patients, so for the group as a whole this type of manipulation of the model does not result in a greater correspondence between observed and predicted ERG voltages than is shown in Fig. 5. In one of these calculations the central annulus was weighted so that it included the area of the blind spot, which (Table 2) is not the case for the results shown in the figures. Since the retinal area involved is so small, this has no effect on the trend of the results.

In the version of the model the results of which are shown in Figs. 5 and 6 only reduction of scotopic sensitivity was considered. In some cases the threshold elevation to the green test light was larger than $2 \log$ units, but since measurements with the red light went off scale it was impossible to tell whether the rods or cones were detecting the green stimulus. These larger values could be incorporated into the model, but such a modification did not cause significant changes to the predictions. If the threshold for a particular retinal region is grossly raised, the ERG voltage contribution for even the stronger flashes is reduced, and swamped by the larger ERGs produced by less severely affected parts of the retina. Thus, for the blue light intensity ranges employed, the ERG amplitude as a function of light intensity is fairly insensitive to changes in assumption $c$. The perimetric determinations were designed to be representative of the visual field as a whole, but in individual cases this might not be so. However, the results show that there is frequently a larger loss of psychophysical sensitivity than of ERG 'sensitivity,' but the reverse is not seen: 


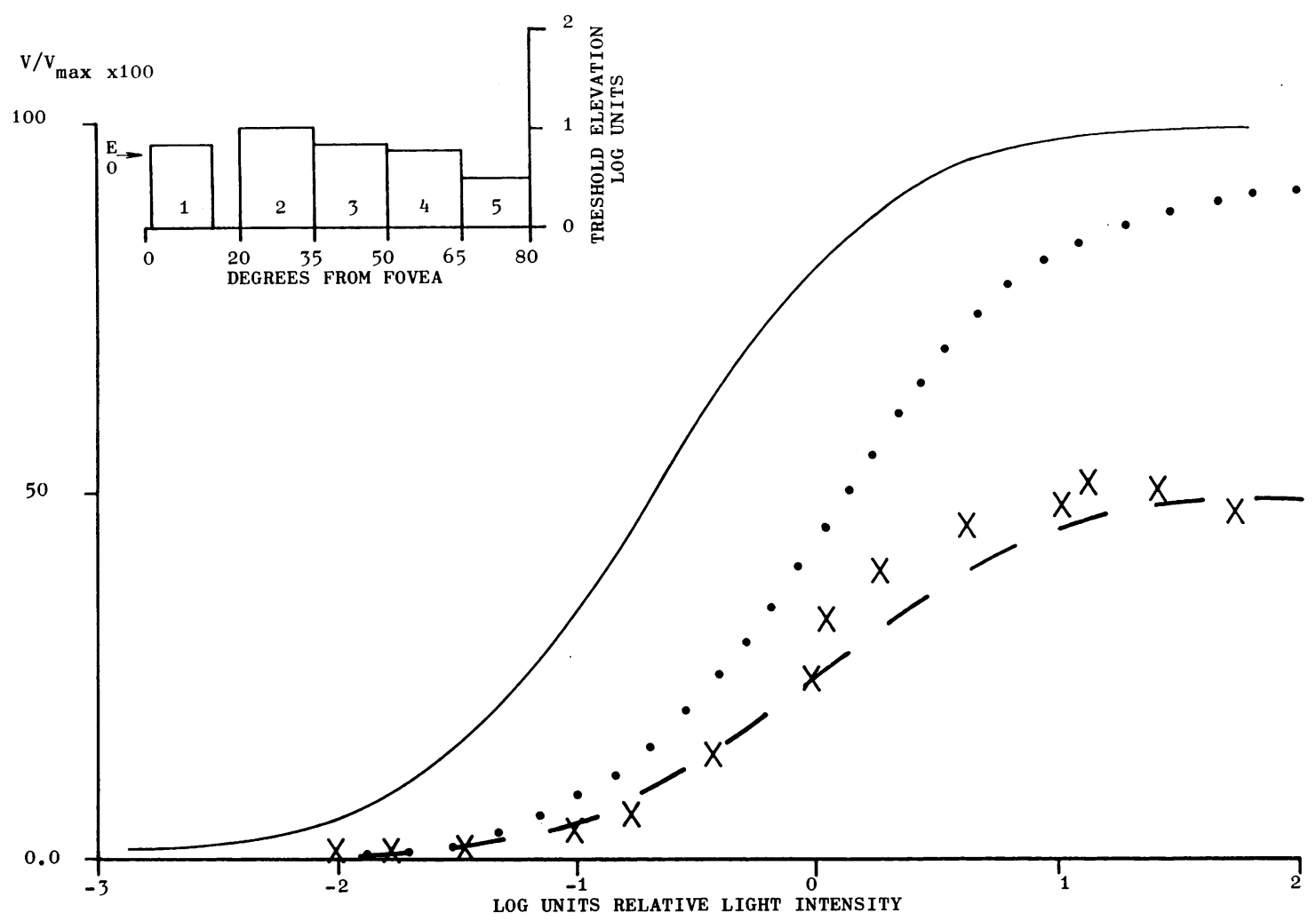

Fig. 8 Patient 10822, female aged 65. Conventions as in Fig. 7. The predicted $b$ wave amplitude was double that recorded. In this and later figures the heavy dashed curve is the model prediction, scaled to agree with the experimental values at higher intensities. This shows that the predicted value of ' $K$ ' for this patient agreed with the experimentally determined value $(0 \cdot 7 \mathrm{log}$ unit). Note that threshold is mildly elevated throughout the visual field.

it is unlikely that the perimeter test points were by chance always in the least sensitive retinal regions. A consideration of the results in individual patients demonstrates how difficult it is to explain the results of Fig. 6 in terms of patient error or peculiarities in the visual field loss.

\section{RESULTS IN INDIVIDUAL PATIENTS}

Fig. 7 inset shows the average threshold elevations in the 5 annuli into which the retina is split for calculation. This patient has constricted visual fields, as is indicated by the high thresholds in the peripheral portions of the retina. Because of this the ERG should be reduced in amplitude. The continuous line in the main figure shows the $\mathrm{V} / \mathrm{V}_{\max }$ curve of the normal scaled as a percentage, where $100 \%$ is equal to $398 \mu \mathrm{V}$. The dotted curve shows the model's prediction. The crosses show the ERG results actually obtained. Note that no adjustment is made for $b$ wave amplitude. The ' $K$ ' value for the patient is slightly lower than predicted, 0.5 against $0.8 \mathrm{log}$ unit. This is within the imprecisions of measurement. The observed $(\mathrm{O})$ and expected $(\mathrm{E})$ values of ' $\mathrm{K}$ ' are indicated in the inset by the arrows. These permit the reader to judge how the calculations correspond to the psychophysical measurements. In this patient the agreement between the ERG data and the model suggests that the assumptions are correct to a first approximation, but such agreement was not found in other patients.

Fig. 8 shows the results from another patient, in whom the threshold elevation was fairly uniform across the visual field. No points on any of the 4 meridia measured gave threshold elevations greater than $2 \log$ units, and the model predicts that with sufficiently strong light a large ERG will be evoked (dotted line). The crosses show the ERGs actually seen which are pathologically reduced in amplitude. If the amplitude of the ERG predicted is reduced (scaled down) so that $\mathrm{V}_{\max }$ agrees with the experimentally determined value, then the dashed curve results, which fits the data obtained, showing that the model's value of ' $K$ ' is correct, even though the estimate of $\mathrm{V}_{\max }$ is not. 
The discrepancy between the predicted and actual ERG voltages in Fig. 8 could be explained if the patient's visual fields were constricted, but there is no evidence that this is the case. Alternatively, if many small randomly distributed patches of retina were affected by the condition, and produced either a reduced or absent $b$ wave, the fields might appear to be full though the ERG was reduced. A similar explanation was advanced to explain the results in $\mathrm{X}$-linked heterozygotes. ${ }^{12}$ The ERG might be supposed to originate from one portion of the retina-for example, the extreme periphery-so the weightings in the model accounted for the discrepancy. However, there is no evidence to support such an assumption, and if it is made it becomes difficult to account for results such as Fig. 7, where there is a peripheral scotoma, and yet the model predictions agree well with observations (see above, 'Modifications to the model').

It was less usual to find ERGs larger than predicted by the model. Fig. 9 shows one such result. The patient is severely affected and has a partial ring scotoma. The ERG recorded (crosses) is larger than predicted (dots). However, the elevation of threshold in static perimetry is so great that the predicted light intensity for $V_{\max }$ is greater than that obtainable with the stimulator, and this explains part of the discrepancy. Additionally, in normal subjects there is a variation of more than $100 \%$ in $\mathrm{V}_{\max }$, so that no special explanation for the size of the ERG may be needed. However, the variation in ' $\mathrm{K}$ ' in normals is less than the difference between the model's prediction and the patient's results. ${ }^{12}$ The dashes show the data scaled so the predicted and observed $V_{\max }$ values agree. This permits the divergence between the observed and expected values of ' $K$ ' to be easily appreciated, and it can be seen that the elevation of ' $\mathrm{K}$ ' is less than predicted. The inset shows that in all the annuli the psychophysical rod threshold was considerably elevated: it is unlikely that considerable portions of the retina of this patient have nearly normal thresholds.

Fig. 10 shows an even more extreme result. This patient had Goldmann fields which were restricted to $15^{\circ}$ eccentricity and very elevated thresholds over the entire visual field. The model predicts a reduced ERG with a much raised ' $K$ ' value. In fact the ERG is almost normal in both respects analysed. There is no

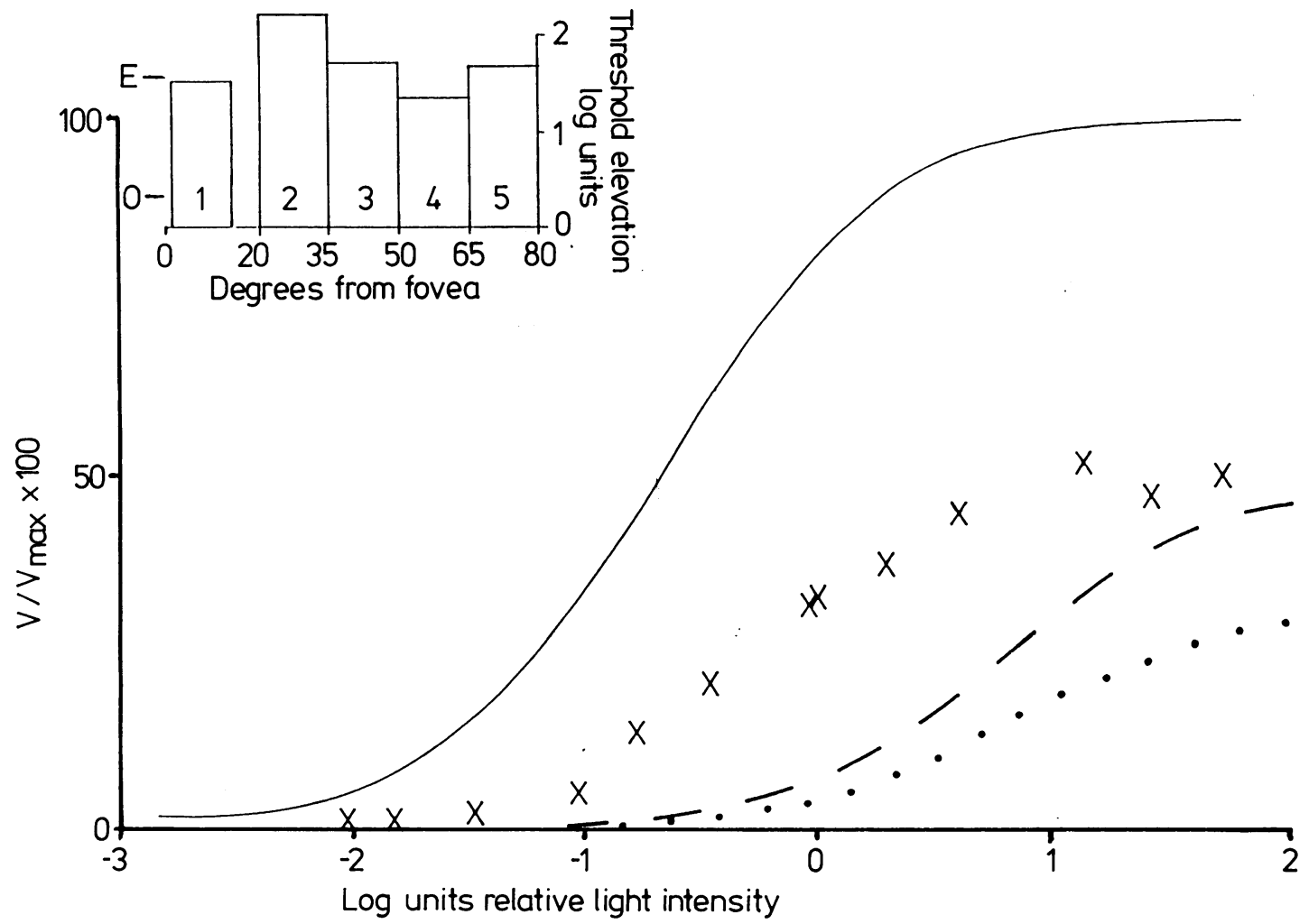

Fig. 9 Patient 10822, female aged 37. Conventions as Fig. 8. Maximum ERG voltage $100 \mu \mathrm{V}$. Note the uniform field losses, which gave a predicted ERG ' $K$ ' value change of $1 \cdot 5 \log$ units, while the measured loss is only $0 \cdot 3 \log$ unit. 


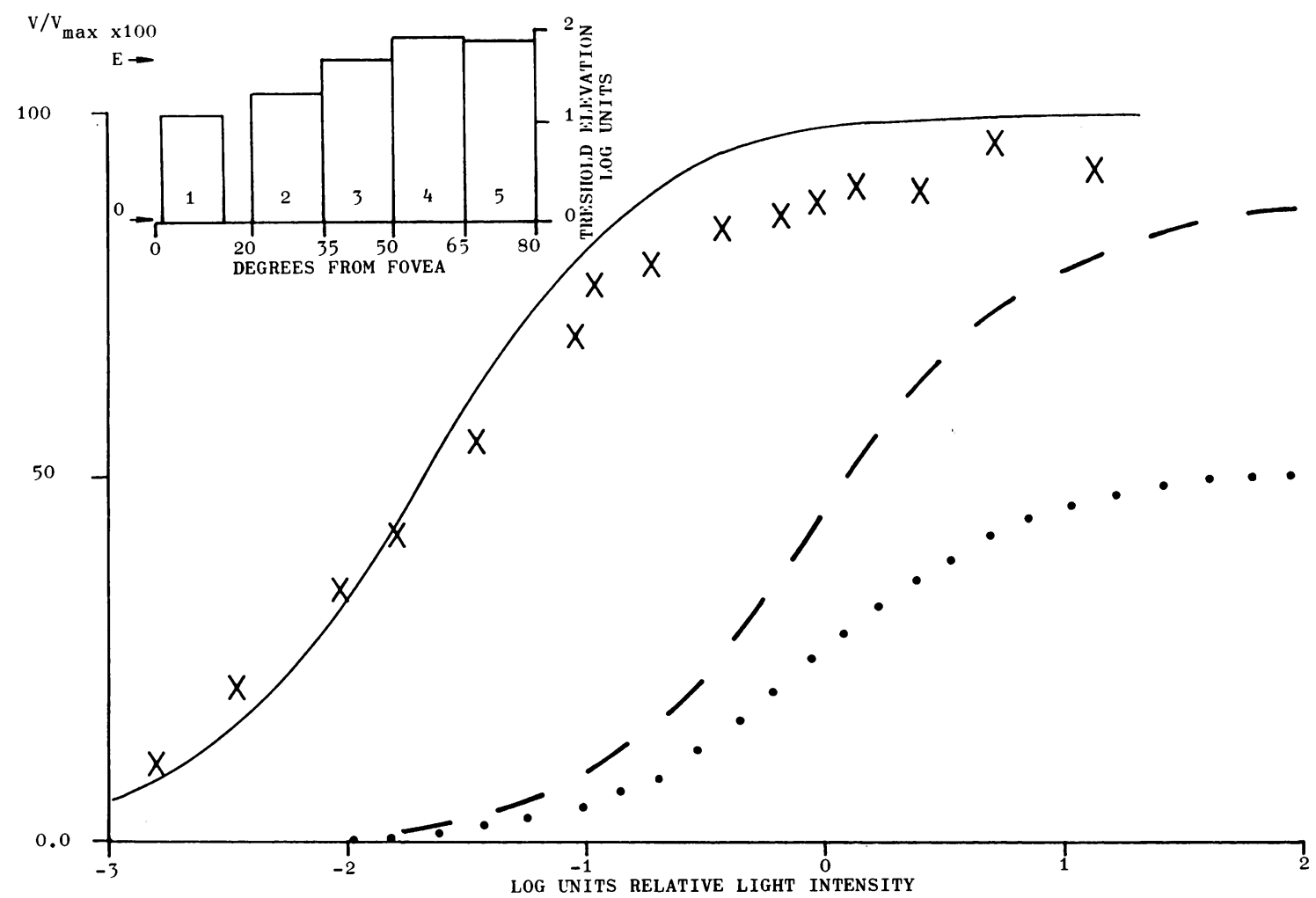

Fig. 10 Patient 10351, female aged 30. Conventions as in Fig. 9. Note loss of sensitivity follows a pattern similar to that shown in Fig. 7, but the ERG is much larger, and the predicted increase of ' $K$ ' is $1 \cdot 7$ log units compared with the ERG measurement of 0 log unit.

sign in this patient that the field losses are due to processes such as optic nerve disease or inner retinal atrophy which might conceivably affect vision without reducing the ERG.

\section{Discussion}

USES OF THE MODEL

In this paper we have used a modified ERG technique to analyse separately the magnitude and sensitivity of the rod signals which lead to the generation of the $b$ wave. In order to make the results more quantitative we have produced a model which relates ERG and psychophysical data. Since the assumptions of the model are explicit, it can be used to predict the ERGs which would occur if the retina was defective in precisely defined ways, and we can determine whether the changes in the ERG seen in the patients are consistent with hypotheses about the nature of the pathological processes which occur in RP. The model is simplified: if visual thresholds were known for every point on the retina and if the relative magnitude of the $b$ wave per unit retinal area was known as a function of retinal location, the model would be improved. Even in its present form it can demonstrate trends in the results so that different subgroups of patients can be distinguished.

\section{SUBDIVISION OF PATIENTS WITH DOMINANT RP}

The ERG results distinguish between patients who do and those who do not have rod $b$ waves. There have been previous attempts to subdivide patients with autosomal dominant RP according to the preservation of rod vision. ${ }^{67}$ Class I has been defined to include those patients in whom there is an early and disproportionate loss of rod function, so that in most, if not all, areas of functional retina vision is entirely mediated by cones. In class II rod and cone vision is affected to approximately the same degree. The present group $B$ includes only class II, but group $A$ contains a mixture of classes I and II. Closer analysis of the results and use of the model suggest reasons for this and provide additional means of subdividing the patient population.

Amplitude of the scotopic $b$ wave. Results from some individual patients (e.g., Figs. 1 and 2) strongly 
suggest that rod ERGs should be recorded when they are not. The model additionally shows that some patients in both the $A_{2}$ and $B$ groups share the property that the rod ERGs are smaller than predicted. It is possible that such patients form a separate subgroup, with a different type of disease. If in patients in whom the amplitude of the rod ERG is overestimated the rod field is small, the scotopic b wave would appear to be extinguished, thus accounting for the $A_{2}$ group. Statistical analysis suggests that groups $A_{1}$ and $A_{2}$ may differ in their properties, and thus $A_{1}$ could correspond to class I, in which there is selective loss of rod sensitivity. Class II could be split into those cases where the rod ERG was predicted by a simple model (including some cases of group $B$ ) and those in which the ERG was disproportionately reduced (the remainder of $B$ and $A_{2}$ ). Thus the presence or absence of rod ERGs may supplement psychophysical testing in the categorisation of patients with dominant RP.

Rod ERGs and the parameter ' $K$ '. Even in those autosomal dominant RP patients who are mildly affected it is common to find a considerable elevation of rod threshold. Thus dark-adapted spectral sensitivity measurements in RP patients ${ }^{14}$ showed a loss of sensitivity of at least 1 log unit compared with normal observers. By contrast, the ERG ' $K$ ' values are little changed in some of our group $B$ patients, though the perimetric tests show they are moderately night blind, and the model predicts ' $\mathrm{K}$ ' values for the ERG which are elevated (Figs. 9 and 10). Thus the degree of correspondence between the ERG and psychophysical measurements of the extent of night blindness may provide yet another way of distinguishing between disorders within the category of autosomal dominant RP, type $B$.

\section{COMMENTS ON THE DISEASE PROCESSES IN RP}

Relationship to fundus reflectometry. The patients with little or no shift in ' $K$ ' values must have some rod photoreceptors which absorb the same proportion of incident light as in normal people, and the rod signals must be of normal size. It is interesting that so large a proportion of the autosomal dominant patients fall into this class. It has recently been shown that a number of patients with RP have considerable quantities of rhodopsin in their retinas ${ }^{89}{ }^{16}$ : the results of Fig. 6 would be inexplicable if this were not to be the case. The results suggest that normal or nearly normal rhodopsin content will be found in patients with dominant RP who are clinically partially night blind. It is possible that patients with large quantities of rhodopsin may form a uniform group, with a different prognosis. It is easier to determine the value of ' $\mathrm{K}$ ' than to measure rhodopsin concentration if it is found that such measurements have clinical significance.
' $K$ ' values and patchy disease. There seems to be a discrepancy between the nearly normal ERG ' $K$ ' value and the partial nightblindness, which could be explained in one of several ways. If, as postulated for heterozygotes for X-linked RP, the disease was patchy, ${ }^{12}$ the affected portions of the retina could develop no ERG, while the perimeter tested the average sensitivity of normal and abnormal regions. However, this seems unlikely because the perimeter test object subtended $1^{\circ}$, while the $b$ wave summation area is considerably larger. A normal ' $\mathrm{K}$ ' value could not be found if the patches of normal retina were smaller than the summation area. ${ }^{12}$

\section{ARE CELLS WHICH ARE NOT PHOTORECEPTORS AFFECTED EARLY IN RP?}

Reduced $b$ wave amplitudes. The $\mathrm{b}$ wave increases in amplitude with increase of light intensity because more of the photoreceptors absorb one quantum each. ${ }^{12}$ However, the maximal rod signal is produced only when 300 or more quanta are absorbed within a short space of time. If the signal from each rod or the proportion of rods which can produce a signal is reduced in disease, ' $K$ ' will increase, but $V_{\max }$ need not change, since fewer, or more weakly responding rods could easily produce a signal which was sufficient to evoke a maximum $b$ wave. This is not what is found. In group $A$ no rod response is recordable, but in $B$ the most common result is that $V_{\max }$ is reduced and ' $K$ ' values are surprisingly close to the normal. Therefore one must make an additional assumption about the nature of the disease process. One possibility is that the disease affects small patches of retina, as discussed above. However, this assumption fails to explain why ' $K$ ' values are raised in some patients and why, in others, abnormal waveforms can be seen. ${ }^{13}$ These findings imply that diseased areas of retina do develop an ERG.

It is possible to make ad hoc assumption about the way in which photoreceptors are affected by the disease. For example, the rods might become noisy, so that signals which adapt bipolar cells develop in darkness, but these do not affect the mechanism which generates the $b$ wave. ${ }^{17}$ An alternative is that the $b$ wave current generators could be reduced in magnitude, or the current pathways modified. In both these cases, the effect of RP on the ERG would be due to damage to cells which are not photoreceptors. The loss of perceptual sensitivity which cannot be seen in the ERG may also be explained if, at some stage subsequent to the neuronal activity which in the end generates the b wave, there is a disturbance of function. Such a phenomenon occurs in the mutant mouse Pearl. ${ }^{18}$ If this is so, RP must be manifest very early at an interneuronal level. Thus, the finding of nearly normal values of ' $K$ ' is important 
in the consideration of the cellular abnormality involved in RP and the search for appropriate animal models.

This work was done with the financial assistance of the American Retinitis Pigmentosa Foundation and the British Retinitis Pigmentosa Society, and the Medical Research Council, to whom we express our gratitude. The work was made possible by the interest and co-operation of Mr B. Jay, Mrs M. Jay, and Professor A. C. Bird, and we thank them for their continuing help.

\section{References}

1 Berson E. Electrical phenomena in the retina. In: Moses RA, ed. Adler's physiology of the eye. New York: Mosby, 1975.

2 Berson EL, Gouras P, Gunkel RD. Rod responses in retinitis pigmentosa dominantly inherited. Arch Ophthalmol 1968; 80: 58-67.

3 Berson EL, Gouras P, Gunkel RD, Myrianthopolous NC. Dominant retinitis pigmentosa with reduced penetrance. Arch Ophthalmol 1969; 81: 226-34.

4 Goodman G. Night-blinding anomalies and diseases. In: Beard C, et al. Congenital anomalies of the eye. Trans $N$ Orleans Acad Ophthalmol, New York: Mosby, 1968: 295-321.

5 Krill A. Hereditary retinal and choroidal diseases. Vols. I and II Hagerstown: Harper and Row, 1972: 1: 189-276; 2: 421-644.

6 Massof RW, Finklestein D. Rod sensitivity relative to cone sensitivity in RP. Invest Ophthalmol Visual Sci 1979; 18: 263 (ARVO abstracts).
7 Massof RW, Finkelstein D. Two forms of autosomal dominant primary retinitis pigmentosa. Doc Ophthalmol 1981; 51:289-346.

8 Highman VN, Weale RA. Rhodopsin density and visual threshold in retinitis pigmentosa. Am J Ophthalmol 1973; 75: 822-32.

9 Ripps H, Brin KP, Weale RA. Rhodopsin and visual threshold in RP. Invest Ophthalmol Visual Sci 1978; 17: 735-45.

10 Ernst W, Clover G, Faulkner DG. X-linked retinitis pigmentosa: reduced rod sensitivity to flicker in heterozygous females. Invest Ophthalmol Visual Sci 1981; 20: 812-6.

11 Fulton AH, Rushton WAH. The human rod ERG correlation with psychophysical responses in light and dark adaptation. Vision Res 1978; 18: 793-800.

12 Arden GB, Carter RM. Ernest WJK, et al. (1982) A modified ERG technique and the results obtained in X-linked retinitis pigmentosa. Br J Ophthalmol 1983; 67: 419-430.

13 Ernst WJK, et al. (submitted to BJO)

14 Massof RW, Johnson MA, Finkelstein D. Peripheral absolute threshold spectral sensitivity in RP. Br J Ophthalmol 1981; 65: 112-21.

15 Drasdo N, Fowler CW. Non-linear projection of the retinal image in a wide angle schematic eye. Br J Ophthalmol 1974; 58: 704-12.

16 Perlman I, Auerbach E. The relationship between visual sensitivity and rhodopsin density in retinitis pigmentosa. Invest Ophthalmol Visual Sci 1981; 20: 758-65.

17 Ashmore JF, Falk G. Photon-like signals following weak rhodopsin bleaches. Nature 1981; 289: 489-91.

18 Balkema LW, Pinto LH, Drager U, Vanable JW. Characterization of abnormalities in the visual system of the mutant mouse Pearl. J Neurosci 1981; 1: 1320-9. 